\title{
Constraints on the symmetry energy and neutron skins from experiments and theory
}

\author{
M. B. Tsang, ${ }^{1}$ J. R. Stone,${ }^{2}$ F. Camera,${ }^{3}$ P. Danielewicz, ${ }^{1}$ S. Gandolfi, ${ }^{4}$ K. Hebeler, ${ }^{5}$ C. J. Horowitz, ${ }^{6}$ Jenny Lee, ${ }^{7}$ W. G. Lynch, ${ }^{1}$ \\ Z. Kohley, ${ }^{1}$ R. Lemmon, ${ }^{8}$ P. Möller, ${ }^{4}$ T. Murakami, ${ }^{9}$ S. Riordan, ${ }^{10}$ X. Roca-Maza, ${ }^{3}$ F. Sammarruca, ${ }^{11}$ A. W. Steiner, ${ }^{12}$ \\ I. Vidaña, ${ }^{13}$ and S. J. Yennello ${ }^{14}$ \\ ${ }^{1}$ National Superconducting Cyclotron Laboratory and Department of Physics and Astronomy, Michigan State University, \\ East Lansing, Michigan 48824, USA \\ ${ }^{2}$ Department of Physics, University of Oxford, OXI 3PU Oxford, United Kingdom and \\ Department of Physics and Astronomy, University of Tennessee, Knoxville, Tennessee 37996, USA \\ ${ }^{3}$ INFN, Sezione di Milano, via Celoria 16, I-20133 Milano, Italy \\ ${ }^{4}$ Theoretical Division, Los Alamos National Laboratory, Los Alamos, New Mexico 87545, USA \\ ${ }^{5}$ Department of Physics, The Ohio State University, Columbus, Ohio 43210, USA \\ ${ }^{6}$ Center for Exploration of Energy and Matter and Physics Department, Indiana University, Bloomington, Indiana 47405, USA \\ ${ }^{7}$ RIKEN Nishina Center, Hirosawa 2-1, Wako, Saitama 351-0198, Japan \\ ${ }^{8}$ Nuclear Physics Group, STFC Daresbury Laboratory, Daresbury, Warrington WA4 4AD, United Kingdom \\ ${ }^{9}$ Department of Physics, Kyoto University, Kyoto 606-8502, Japan \\ ${ }^{10}$ University of Massachusetts Amherst, Amherst, Massachusetts 01003, USA \\ ${ }^{11}$ Physics Department, University of Idaho, Moscow, Idaho 83844-0903, USA \\ ${ }^{12}$ Institute for Nuclear Theory, University of Washington, Seattle, Washington 98195, USA \\ ${ }^{13}$ Centro de Física Computacional, Department of Physics, University of Coimbra, PT-3004-516 Coimbra, Portugal \\ ${ }^{14}$ Cyclotron Institute and Chemistry Department, Texas A\&M University, College Station, Texas 77843, USA
}

(Received 21 March 2012; published 5 July 2012)

\begin{abstract}
The symmetry energy contribution to the nuclear equation of state impacts various phenomena in nuclear astrophysics, nuclear structure, and nuclear reactions. Its determination is a key objective of contemporary nuclear physics, with consequences for the understanding of dense matter within neutron stars. We examine the results of laboratory experiments that have provided initial constraints on the nuclear symmetry energy and on its density dependence at and somewhat below normal nuclear matter density. Even though some of these constraints have been derived from properties of nuclei while others have been derived from the nuclear response to electroweak and hadronic probes, within experimental uncertainties-they are consistent with each other. We also examine the most frequently used theoretical models that predict the symmetry energy and its slope parameter. By comparing existing constraints on the symmetry pressure to theories, we demonstrate how contributions of three-body forces, which are essential ingredients in neutron matter models, can be determined.
\end{abstract}

DOI: 10.1103/PhysRevC.86.015803

PACS number(s): 21.65.Ef, 21.65.Cd, 26.60.Kp, 21.45.Ff

\section{INTRODUCTION}

Contemporary nuclear science aims to understand the properties of strongly interacting bulk matter at the nuclear, hadronic, and partonic levels $[1,2]$. In addition to their contributions in fundamental physics, such studies have enormous impact on astrophysics, from the evolution of the early universe to neutron star structure [3]. For example, a precise knowledge of the equation of state of neutron matter is essential to understand the physics of neutron stars and binary mergers, predicted to be strong sources of gravitational waves [4]. While the equation of state of symmetric nuclear matter consisting of equal amount of neutrons and protons has been constrained over a wide range of densities [5], our knowledge on asymmetric nuclear matter is very limited, largely as a consequence of our inadequate understanding of the symmetry energy $[5,6]$. The symmetry energy describes the response of the force on protons and neutrons in a nuclear system with excess neutrons or protons. It reduces the nuclear binding energy in nuclei with $N \neq Z$ and is critical for understanding the properties of nuclei, including the existence of rare isotopes with extreme proton to neutron ratios [7-9].
Its slope parameter at saturation density provides the dominant baryonic contribution to the pressure in neutron stars [10] and affects the neutron skin thicknesses in heavy nuclei. Knowledge of the neutron skin thickness is important for atomic parity violation (APV) experiments because atomic electrons have weak interactions, primarily with neutrons (see, for example, [11-13]). Therefore, constraining the neutron skin thickness may be important for low-energy APV tests of the standard model. Conversely, one can measure APV for a range of isotopes to deduce neutron radii [14].

For all its importance, we do not have a realistic model of nuclear forces that can describe the equation of state of neutron matter [15-29] over a wide range of densities. Recently, substantial progress in our understanding of the symmetry energy has been made both experimentally [30-54] and theoretically [15-29,55,56], in particular at subsaturation densities. Astrophysical observations of neutron stars are also starting to provide quantitative constraint to the symmetry energy [57,58]. Aside from describing the progress on symmetry energy and the neutron skin thickness in ${ }^{208} \mathrm{~Pb}$, we will discuss how the results can be used to constrain theoretical calculations and to test the 


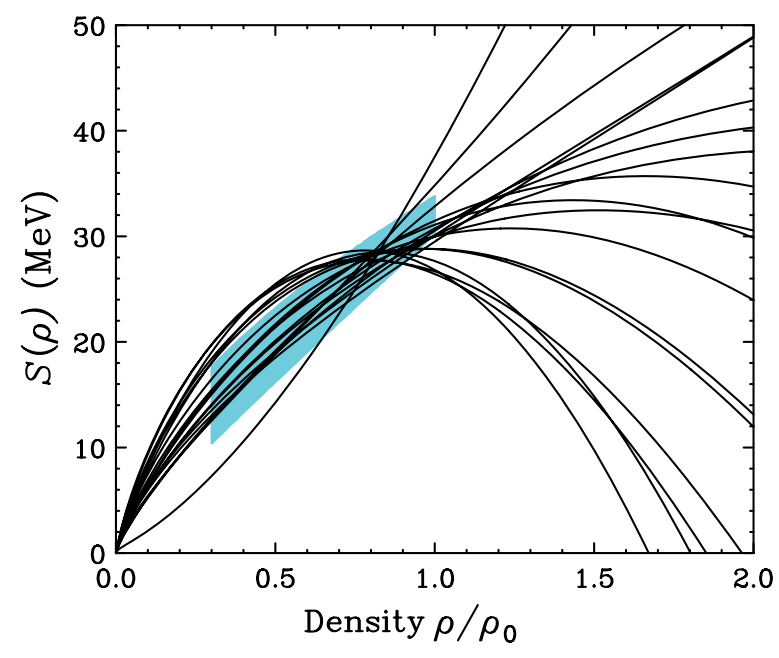

FIG. 1. (Color online) Density dependence of the symmetry energy from the Skyrme interactions used in Ref. [6]. The shaded region is obtained from HIC experiments as described in the text and corresponds to the shaded region in Fig. 2.

importance of three-body and higher order forces in microscopic models.

Using the Skyrme-Hartree-Fock model, Brown [6] pointed out a decade ago that selected Skyrme parameterizations, which fit the binding energy difference between ${ }^{100} \mathrm{Sn}$ and ${ }^{132} \mathrm{Sn}$ nuclei, may predict very different density dependencies of the energy per nucleon in pure neutron matter at densities above and below saturation density. Figure 1 shows that the symmetry energy, which represents the difference between the energies of symmetric and pure neutron matter, is equally uncertain. Brown also discovered a nearly linear correlation between the neutron skin thickness in heavy nuclei and the pressure of the neutron matter equation of state (EOS) at $\rho \approx 0.625 \rho_{0}$, a trend replicated later by relativistic Hartree model calculations [59]. Many observables, from nuclear masses to nuclear structure and nuclear dynamics, also display significant sensitivities to the density dependence of the symmetry energy in the region near saturation density and below $\left(0.3 \leqslant \rho / \rho_{0} \leqslant 1\right)$. Since the constraints obtained from heavy ion collisions (HICs) have been compared to constraints obtained from pygmy dipole resonance (PDR) and the isobaric analog states in Ref. [30], many new results become available. In this article, we are able to update the PDR constraints as well as to include constraints from nuclear masses and from the elastic scattering of ${ }^{208} \mathrm{~Pb}$. A constraint on the symmetry energy obtained from determination of neutron star radius is also included in our compilation.

To the lowest order expansion, the EOS of cold nuclear matter can be approximately written as the sum of the energy per nucleon of symmetric matter and an asymmetry term $[5,55,56]$

$$
E(\rho, \delta)=E_{0}(\rho, \delta=0)+S(\rho) \delta^{2},
$$

where $\delta=\left(\rho_{n}-\rho_{p}\right) / \rho$ is the asymmetry, and $\rho_{n}, \rho_{p}$, and $\rho$ are the neutron, proton, and nucleon densities, respectively. (For simplicity, we do not include the readily calculated electromagnetic contribution to the energy.) The symmetry energy factor, $S(\rho)$ depends on density.

It is useful to expand the symmetry energy factor $S(\rho)$ in Eq. (1) in a Taylor series around the saturation density, $\rho_{0} \sim$ $0.16 \mathrm{fm}^{-3}$, where the binding energy of symmetric nuclear matter reaches its maximum value of $\sim 16 \mathrm{MeV}$ :

$$
S(\rho)=S_{0}-L \varepsilon+1 / 2 K_{\mathrm{sym}} \varepsilon^{2}+O\left[\varepsilon^{3}\right],
$$

where $\varepsilon \equiv\left(\rho_{0}-\rho\right) /\left(3 \rho_{0}\right)$. In the literature, $S_{0}$ is sometimes also denoted as $J . L$ and $K_{\text {sym }}$ are the slope and curvature parameters at $\rho_{0}$. The slope parameter $L$,

$$
L=3 \rho_{0} d S(\rho) /\left.d \rho\right|_{\rho_{0}}=\left[3 / \rho_{0}\right] P_{0},
$$

governs $P_{0}$, the pressure from the symmetry energy in pure neutron matter at $\rho_{0} . P_{0}$ provides the dominant baryonic contribution to the pressure in neutron stars at $\rho_{0}[10]$ and influences the inner crusts and radii of neutron stars $[3,60]$. Thus $L$ forms an essential link between nuclear physics and astrophysics [10].

Realistic models of nuclear matter and its effective interactions predict model-dependent correlations between $S_{0}, L$, and $K_{\text {sym }}$. Observables with different sensitivities to $S_{0}, L$, and $K_{\text {sym }}$ can be combined to allow independent constraints on them and on the theories from which they can be calculated. However, $K_{\text {sym }}$ correlates strongly with $L$ and contributes weakly to the symmetry energy at subsaturation densities, making it difficult to constrain $K_{\text {sym }}$ [61]. In the following, we discuss constraints on $S_{0}$ and $L$ extracted from ground-state properties, such as nuclear masses and neutron skin thicknesses and from excited-state properties, such as the energies of isobaric analog states, and the energies and strengths of giant and pygmy dipole resonances. We also discuss constraints provided by observables sensitive to the transport of neutrons and protons during nucleus-nucleus collisions. In addition, we compile results from recent measurements of the neutron skin thickness of ${ }^{208} \mathrm{~Pb}$, the best candidate for precise neutron skin measurement. We examine critically the consistency between different measurements, including experimental and theoretical uncertainties, and discuss the ability of future experiments to provide further constraints.

We note that these constraints on $S_{0}$ and $L$ are most directly applicable to matter at uniform or nearly uniform density. Low-density $\rho<0.3 \rho_{0}$ matter plays important roles in neutron stars and core collapse supernovae and is not uniform [62]; consequently, the mean field does not directly apply. Laboratory experiments have been performed to investigate the properties of low-density nuclear matter. We refer readers to the work of Natowitz et al. [63] for a recent exploration of the interplay of clusterization and the low-density symmetry energy in laboratory systems. The constraints on $S_{0}$ and $L$ discussed in this paper can be relevant to statistical models that describe the separation of matter into dilute and dense phases, where the latter resemble nuclei [64].

This paper is organized as follows. Recent experimental measurements providing constraints on the nuclear symmetry energy and the neutron skin thickness of ${ }^{208} \mathrm{~Pb}$ are discussed in Sec. II. Discussion of the effects of the constraints on different theoretical models is presented in Sec. III, followed by summary and outlook in Sec. IV. 


\section{EXPERIMENTAL CONSTRAINTS ON THE SYMMETRY ENERGY}

\section{A. Symmetry energy constraints from heavy ion collisions (HIC)}

Large density variations can be attained for a very short period of time in HIC using projectiles such as $\mathrm{Au}$ or $\mathrm{Sn}$ $[5,55,56]$. The EOS is an essential input to the transport models and can be constrained by comparing measurements of such collisions to transport model calculations $[5,55,56,65,66]$. This strategy was successfully applied to constrain the EOS of symmetric matter, $E_{0}(\rho, \delta=0)$, at densities of $\rho_{0} \leqslant \rho \leqslant$ $5 \rho_{0}$, by studying energetic ${ }^{197} \mathrm{Au}+{ }^{197} \mathrm{Au}$ collisions $[5,31,65]$.

To gain sensitivity to the symmetry energy and extrapolate to neutron-rich asymmetric matter, one can vary the neutron and proton numbers of the projectile and target nuclei so as to compare emission of particles from neutron-rich systems to that from neutron-deficient systems [30-40]. The influence of the symmetry potential can be more easily distinguished from other effects by comparing the emitted nucleons and light nuclei with different neutron and proton numbers. Especially interesting are the comparisons between "mirror" nuclear ejectiles with the same mass, and total isospin, but where the proton and neutron numbers are exchanged, e.g., comparing the emission of neutrons to protons, ${ }^{3} \mathrm{H}$ to ${ }^{3} \mathrm{He},{ }^{7} \mathrm{Li}$ to ${ }^{7} \mathrm{Be}$ $[31,34,36,55,56]$. Such comparisons probe the combination of Coulomb and symmetry mean-field potentials. The latter has the opposite sign for protons and neutrons, but the combination contributes greatly to the uncertainty in the symmetry energy [55].

In a neutron-rich environment, the symmetry potential tends to expel neutrons and attract protons, enhancing the yield ratios of ejected neutrons/protons and other isotopes while influencing their dependence on the ejected particle's momentum $[34,55,56]$. Neutron-proton spectral ratios [34] and neutron, hydrogen [35], and fragment flows [36,37] have all been used to study the density dependence of the symmetry energy. When the collision involves projectiles and targets of different $N / Z$ asymmetry, and different local density, the symmetry potential pushes the system towards an "isospin equilibrium" characterized by constant values of $\delta=\left(\rho_{n}-\rho_{p}\right) / \rho$, throughout the system. Thus, the magnitude of the symmetry potential in a low-density "neck" region joining a projectile and target during a peripheral or midcentral collision governs the rate of "isospin diffusion" between a projectile and a target of different asymmetry. This phenomenon has been used to probe the symmetry energy $[32,33,38-40,55]$.

The neutron and proton spectra from central collisions for ${ }^{124} \mathrm{Sn}+{ }^{124} \mathrm{Sn}$ and ${ }^{112} \mathrm{Sn}+{ }^{112} \mathrm{Sn}$ collisions at $50 \mathrm{MeV}$ per nucleon [34] have been measured. At the same incident energy, isospin diffusion was investigated. Normalization of the latter requires asymmetric systems ${ }^{124} \mathrm{Sn}+{ }^{112} \mathrm{Sn}\left({ }^{112} \mathrm{Sn}+{ }^{124} \mathrm{Sn}\right)$ to be compared to symmetric systems of ${ }^{124} \mathrm{Sn}+{ }^{124} \mathrm{Sn}$ and ${ }^{112} \mathrm{Sn}+{ }^{112} \mathrm{Sn}$ collisions $[33,39,40]$. A $\chi^{2}$ analysis compared, measured, and calculated values for the ratios of neutron-toproton spectra as well as two observables sensitive to isospin diffusion [30]. In Fig. 2, a set of constraints within two standard deviations from the minimum, corresponding to $95 \%$ confidence levels, is shown as a shaded band bounded by

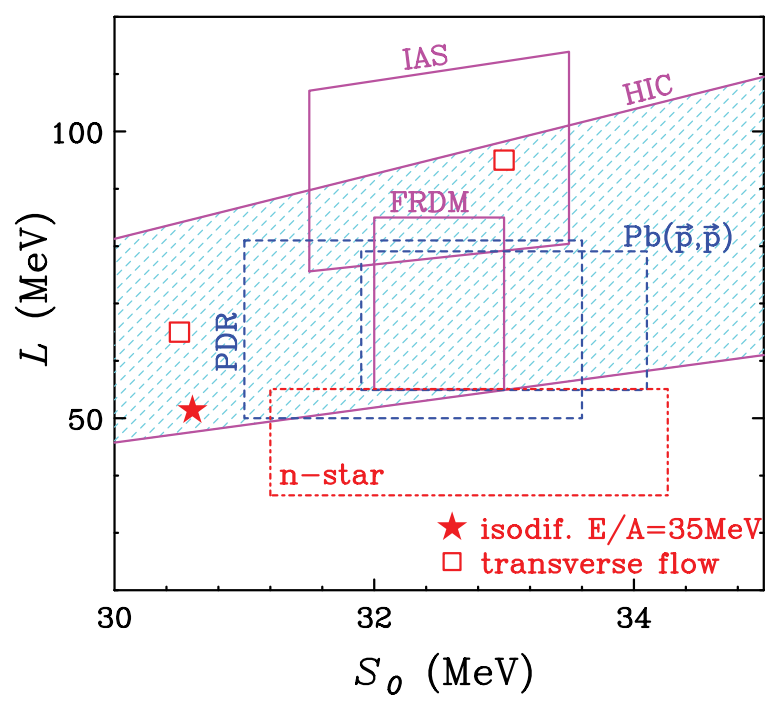

FIG. 2. (Color online) Constraints on the slope $L$ and magnitude $S_{0}$ of the symmetry energy at saturation density from different experiments. The experimental methods are labeled next to the boxes with the estimated uncertainties. The symbols are results without the analysis of the errors. See text for details.

two diagonal lines in the $\left(L, S_{0}\right)$ plane. The solid star shows the results from isospin diffusion observables measured for collisions at a lower beam energy of $35 \mathrm{MeV}$ per nucleon [40]. The corresponding density dependence of the symmetry energy is plotted as the shaded region in Fig. 1. All observables obtained from the sets of data described here were mainly sensitive to the symmetry energy at densities $0.3 \rho_{0} \leqslant \rho \leqslant \rho_{0}$.

Transverse collective flows of hydrogen and helium isotopes [36,37] as well as intermediate mass fragments with $Z<9$ have also been measured at incident energy at $35 \mathrm{MeV}$ per nucleon in ${ }^{70} \mathrm{Zn}+{ }^{70} \mathrm{Zn},{ }^{64} \mathrm{Zn}+{ }^{64} \mathrm{Zn}$, and ${ }^{64} \mathrm{Ni}+{ }^{64} \mathrm{Ni}$ collisions and compared to transport calculations. These comparisons yielded values for $\left(S_{0}, L\right)$ denoted by the open squares in Fig. 2. No extensive $\chi^{2}$ or sensitivity analyses have been performed over the $\left(S_{0}, L\right)$ space for either the transverse flow data (open squares) or the low-energy isospin diffusion data (solid star). We consequently plot them without error bars.

\section{B. Symmetry energy constraints from nuclear binding energies}

In 1935, Bethe and Weizäcker et al. proposed a very successful theory of nuclear binding energies $[7,8]$. To a good approximation, this theory reduces to a remarkably simple mass formula in which the binding energy $B(N, Z)$ is obtained as a function of proton number $Z$ and neutron number $N$, with mass number, $A=N+Z$,

$$
\begin{aligned}
B(N, Z)= & a_{\mathrm{vol}} A-a_{\mathrm{surf}} A^{2 / 3}-a_{C} \frac{Z^{2}}{A^{1 / 3}} \\
& -a_{\mathrm{sym}} \frac{(N-Z)^{2}}{A}+d E,
\end{aligned}
$$

in terms of volume, surface, Coulomb, symmetry energies, and additional small contributions related to microscopic effects 
$[7,8]$. The coefficients in Eq. (4) can be determined by fitting to known atomic masses across the nuclear chart.

Myers and Swiatecki separated the volume and surface contributions to the symmetry energy in their liquid drop model [67] and subsequently developed a refined version of the model, called the droplet model, by expanding the volume, surface, and Coulomb energies in a Taylor series in terms of $(N-Z) / A$ and $A^{-1 / 3}$ around the standard liquid drop model values. This introduced additional degrees of freedom allowing for the deviations from uniformity of the proton and neutron densities and led to a more realistic parameterization of the symmetry energy [67].

Since the symmetry energy contribution to the total binding energy can be small relative to those from the other terms, the unambiguous determination of $S_{0}$ and $L$, the magnitude and slope of the symmetry energy at saturation density, has proven difficult $[60,68]$. To overcome the problem, all main contributions to the binding energy must be theoretically described with the highest possible accuracy. A refinement of the droplet model, the finite-range droplet model (FRDM) [9], came close to fulfillment of this requirement. It included additional important features such as microscopic "shell" effects and the extra binding associated with $N=Z$ nuclei. The FRDM reproduced binding energies of known nuclei with a deviation $\sigma=0.67 \mathrm{MeV}$ and implied a value of $S_{0}=$ 32.73 MeV [9].

Despite the greatly improved predictions for nuclear binding energies it provided, it still did not have enough sensitivity to determine $L$. More complex calculations [9] that include additional effects such as axially asymmetric nuclear groundstate shapes further improved the deviation of nuclear binding energies to $\sigma=0.57 \mathrm{MeV}$. This means that the nuclear binding energies are reproduced to within $0.1 \%$. The model now allows determination of both $S_{0}=32.5 \pm 0.5 \mathrm{MeV}$ and $L=70 \pm$ $15 \mathrm{MeV}$. This constraint is shown as a square box in Fig. 2, labeled "FRDM" on the top. Although the results are consistent with other model predictions, this very small uncertainty in the value of $S_{0}$, if correct, limits seriously the choice of currently available equations of state used in modeling neutron stars and supernova matter.

\section{Symmetry energy constraints from isobaric analog states}

Fits of nuclear binding energies to mass models must address ambiguities stemming from the similarities in the influences of Coulomb and symmetry energy terms over the range of experimentally measured masses. These ambiguities in the determination of the symmetry energy from binding energies caused by the Coulomb term can be removed by taking advantage of the charge independence of nuclear interactions, i.e., to a very good approximation, strong interactions between nucleons in the same state do not depend on whether the nucleons are protons or neutrons [42,61]. For example, there is an excited state in the nucleus ${ }^{12} \mathrm{C}(Z=N=6)$ with the same wave function and nuclear contribution to the binding energy as the ground state of its isobar, ${ }^{12} \mathrm{~B}(Z=5$, $N=7)$. This ${ }^{12} \mathrm{C}$ excited state is called the isobaric analog state (IAS) of the ground state of ${ }^{12} \mathrm{~B}$, and its binding energy differs from that of the ${ }^{12} \mathrm{~B}$ ground state by the difference in their Coulomb energies. It follows from Eq. (4) that the energy differences between the ground state for a nucleus with $N>Z$ and the isobaric analogs of the ground states of neighboring isobars are given by the symmetry energy since the Coulomb contributions to the binding energy can be determined using the IAS.

To utilize this technique, however, one must realize mathematically that the nuclear Hamiltonian is charge independent to a good approximation. It depends on the total isospin operator $T^{2}$ and not on its projection $T_{z}$ which has the eigenvalue $(N-Z) / 2$ for a nucleus with neutron and proton number $N$ and $Z$, respectively. This can be accomplished by replacing $E_{\text {sym }} \approx a_{\text {sym }}(A) \frac{(N-Z)^{2}}{A}$ with $E_{\text {sym }}=\frac{4 a_{\text {sym }}(A)}{A} T(T+1)$ [42]. Here, we express $a_{\text {sym }}$ as a function of $\mathrm{A}$. This allows the asymmetry coefficient $a_{\text {sym }}(A)$ to be determined on a nucleus-by-nucleus basis from

$$
a_{\mathrm{sym}}(A) \approx-\frac{\Delta B}{4 \Delta T^{2}} A,
$$

where $\Delta T^{2}=T_{\mathrm{IAS}}\left(T_{\mathrm{IAS}}+1\right)-T_{\mathrm{gs}}\left(T_{\mathrm{gs}}+1\right)$ is the difference between the known $T^{2}$ eigenvalues for the isobaric analog states and the ground state in the same nucleus, and $\Delta B$ is the difference in the binding energies of these two states. Many such states have been identified [69]. By fitting the available data on the IAS, Danielewicz and Lee obtained the constraint $[42,61]$ shown as a parallelogram in Fig. 2, labeled IAS inside the box. Further refinements to these fits are in progress [42].

\section{Neutron skin thickness measurements}

In light nuclei with $N \approx Z$, the neutrons and protons have similar density distributions. With increasing neutron number, the radius of the neutron density distribution becomes larger than that of the protons, reflecting the pressure of the symmetry energy. The difference $\delta R_{n p}$ in the neutron and proton rootmean-square radii is called the neutron skin, i.e.,

$$
\delta R_{n p}=\left\langle r^{2}\right\rangle_{n}^{1 / 2}-\left\langle r^{2}\right\rangle_{p}^{1 / 2}
$$

Proton radii have been determined accurately for many nuclei using electron scattering experiments [70]. This accuracy reflects the accuracy of perturbative treatments of the electromagnetic process. The neutron density distribution is more difficult to measure accurately because it interacts mainly with hadronic probes (protons, alphas, pions, and antiprotons) through nonperturbative interactions, the theoretical description of which is model dependent.

The stable nucleus ${ }^{208} \mathrm{~Pb}$ is a very interesting candidate for determining the neutron skin. With a closed neutron shell at $N=126$ and a closed proton shell at $Z=82$, it is very asymmetric with thick neutron skin. The closed shells make its structure relatively well understood, allowing a clean relationship between skin thickness and properties of the symmetry energy. As the same physical mechanisms acting on the neutrons in the lead nucleus also act on the neutrons in the interior of neutron stars, the relationship between the neutron skin thickness of $\sim 0.2 \mathrm{fm}$ and the radius of neutron star of $\sim 12 \mathrm{~km}$, predicted by many models $[6,10,59,60]$, has stimulated many experiments to measure the neutron radius of 
${ }^{208} \mathrm{~Pb}$ [43-50]. We note, however, that the neutron star radius reflects the pressure due to the symmetry energy at a range of densities and is also highly sensitive to its pressure at 2-3 times saturation density [3].

In the following subsections, we discuss experimental measurements that probe neutron skins using both electroweak and hadronic probes. All of these experiments require theoretical models to extract the neutron skin thickness of ${ }^{208} \mathrm{~Pb}$ from the data. There is currently no consistent way to estimate the theoretical errors arising from the models. Furthermore, the models used may vary, often depending on the familiarity of the authors with a certain type of model. However, in the evaluation of the antiprotonic atom data in Ref. [71], Brown et al. gave a rather detailed account of how the theoretical uncertainty of $0.05 \mathrm{fm}$ is deduced. Except for the $\mathrm{Pb}$ radius experiment (PREX) at Jefferson Laboratory and the antiprotonic experiments, the experimental uncertainties are much less than $10 \%$. Without a comprehensive study of the theoretical and experimental uncertainties in the measurements described below, we adopt a minimum error in $\delta R_{n p}$ of $\pm 0.05 \mathrm{fm}$ (a rough estimate of the model uncertainties) in order not to bias comparisons of various $\delta R_{n p}$ values towards values with the most underestimated uncertainties.

\section{Parity violating electron scattering}

The possibility of measurements of the neutron radius in ${ }^{208} \mathrm{~Pb}$ by PREX at Jefferson Laboratory had been widely discussed [72,73]. This experiment is designed to extract the neutron radius in ${ }^{208} \mathrm{~Pb}$ from parity-violating electron scattering. The electroweak probe has the advantage over experiments using hadronic probes in that it allows a nearly model-independent extraction of the neutron radius that is independent of most strong interaction uncertainties [72]. The experimental signature, however, is very small, making high-precision measurements very challenging [73]. Due to technical problems in a recent measurement [43], which reduced the statistics severely, the extracted ${ }^{208} \mathrm{~Pb}$ skin thickness had large statistical uncertainties of $\delta R_{n p}=0.33_{(-0.18)}^{(+0.16)} \mathrm{fm} \mathrm{[43].}$ A second experimental run has now been approved for PREX to run in a few years to achieve the original goal of measuring the skin thickness of ${ }^{208} \mathrm{~Pb}$ with an uncertainty of $\pm 0.05 \mathrm{fm}$ in $\delta R_{n p}$ [74].

\section{Proton elastic scattering of ${ }^{208} \mathrm{~Pb}(\vec{p}, \vec{p})$}

Zenihiro et al. recently reported an extraction of the neutron skin thickness of ${ }^{208} \mathrm{~Pb}$ via polarized proton elastic scattering [44]. Cross sections and vector analyzing powers for polarized proton elastic scattering on ${ }^{58} \mathrm{Ni}$ and ${ }^{204,206,208} \mathrm{~Pb}$ were measured with high precision. A $T$-matrix parameterization of the proton optical potential was constrained by the ${ }^{58} \mathrm{Ni}$ measurements and fit to the ${ }^{204,206,208} \mathrm{~Pb}$ data by adjusting the neutron densities. The deduced neutron skin thickness of ${ }^{208} \mathrm{~Pb}, \delta R_{n p}=0.211_{(-0.063)}^{(+0.054)} \mathrm{fm}$. This uncertainty includes an estimated theoretical uncertainty arising from using different models for the nucleon-nucleon interaction. The symmetry energy constraints consistent with the determined skin thickness of ${ }^{208} \mathrm{~Pb}$ were evaluated within both relativistic and nonrelativistic models and plotted as the dashed blue rectangular box labeled " $\mathrm{Pb}(\vec{p}, \vec{p})$ " in Fig. 2 .

\section{Antiprotonic atoms}

Neutron skins of many other nuclei have been probed by measurements $[47,48]$ of photons emitted during the decays of antiprotonic states of high orbital angular momentum where the angular momentum barrier restricts the interactions between the antiproton and the neutron density to large distances. The root-mean-square neutron radius is not directly measured, making the skin thickness results strongly dependent on theoretical models. Nevertheless, systematic measurements of the neutron skins of a range of nuclei can contribute significantly to a global understanding of the evolution of neutron density distributions with nuclear charge and mass. A systematic analysis of the neutron skin of 26 antiprotonic atoms ranging from ${ }^{40} \mathrm{Ca}$ to ${ }^{238} \mathrm{U}[47,49]$ suggests that there is a correlation between the neutron skin thickness and the isospin asymmetry. The adopted value of $\delta R_{n p}=0.18 \pm(0.04)_{\exp }$ $\pm(0.05)_{\text {theo }}$ fm come from averaging the results of [47-49]. In Ref. [49] a droplet model is used to determine a correlation between $\delta R_{n p}$ and $L$, resulting in the constraints $L=55 \pm 25$ $\mathrm{MeV}$ and $S_{0}=31.5 \pm 2 \mathrm{MeV}$. The uncertainties of the results from the antiproton experiments are larger than those in the other experiments discussed here. Nonetheless, the method of using the systematics of a range of nuclei to extract the skin thicknesses of ${ }^{208} \mathrm{~Pb}$ remains attractive, especially if both the experimental and theoretical uncertainties could be reduced when good quality skin thickness measurements of a wide range of nuclei become available.

\section{Electric dipole strength function}

a. Electric dipole polarizability. The electric dipole polarizability (EDP) $\alpha$ is defined by the relationship $\vec{p}=\alpha \vec{E}$ between the induced electric dipole moment $\vec{p}$ of a nucleus and the static electric field $\vec{E}$ that induces it. While the polarizability of some light nuclei has been determined by placing them in the field of a heavy target nucleus [75], it is more common to excite the nucleus by photoexcitation [76,77] or Coulomb excitation [50] and use the relationship $\alpha=\frac{\hbar c}{2 \pi^{2} e^{2}} \int \frac{\sigma_{\mathrm{ABS}}}{\omega^{2}} d \omega$ between $\alpha$ and the photon absorption cross section $\sigma_{\mathrm{ABS}}$ weighted with $\omega^{-2}$ and integrated over the incident photon energy $\omega$.

The interaction of nuclei with an electric dipole field leads to the real or virtual excitation of nuclear excited states, many of which contribute to the giant dipole resonance (GDR) in nuclei [51]. Semiclassically, the GDR can be viewed as a collective vibration in which neutrons and protons move in opposite directions, displacing the neutron and proton densities relative to each other and increasing the symmetry energy. Thus, the symmetry energy contributes to the "restoring force" for the vibration and strongly influences the excitation energies of states that can be easily excited by an electric field $[77,78]$.

In neutron-rich nuclei a significant enhancement in the EDP can be expected due to the development of a neutron skin. In such nuclei, one has the possibility of vibrations of the $(N, Z)$ symmetric core against the neutrons in the skin. This leads to the appearance of low-energy states that are easily excited 
by electric dipole radiation, i.e., low-energy dipole strength, which greatly enhances $\alpha$ due to the $\omega^{-2}$ weighting in the integral above. In fact, the low-energy dipole strength can contribute as much as $25 \%$ to the dipole polarizability [79]. For very neutron-rich nuclei, this enhanced dipole strength can be localized in energy and appears in the form of a PDR discussed in the next section.

Reinhard and Nazarewicz predicted a strong correlation between the neutron skin thickness in ${ }^{208} \mathrm{~Pb}$ and the EDP within a model with a Skyrme interaction and an effective Lagrangian [80]. Recent experiments using inelastic scattering of polarized protons on ${ }^{208} \mathrm{~Pb}$ at very forward angles [50] have provided the complete electric dipole response of ${ }^{208} \mathrm{~Pb}$ from low excitation energies up to the GDR with high resolution. A precise value of the EDP was extracted and the calculations from Ref. [80] were used to predict a value for the neutron skin thickness $\delta R_{n p}=0.156_{(-0.021)}^{(+0.025)}$ fm for ${ }^{208} \mathrm{~Pb}$ [50]. More recent calculations have shown the skin thickness results to be somewhat model dependent [81].

b. Pygmy dipole resonances. In very neutron-rich nuclei such as ${ }^{68} \mathrm{Ni}$ and ${ }^{132} \mathrm{Sn}$, enhanced low-energy electric dipole strength has been observed and attributed to a PDR that peaks at excitation energies well below the GDR [52-54]. These PDR peaks can exhaust in the order of $5 \%$ of the energy-weighted sum rule (EWSR). In many models, the calculated percentage of the EWSR exhausted by PDR is shown to be linearly related to the slope parameter $L$ of the symmetry energy. Carbone et al. extracted a value of $L=64.8 \pm 15.7 \mathrm{MeV}$ [53] from measurements of the PDR for ${ }^{68} \mathrm{Ni}[54]$ and ${ }^{132} \mathrm{Sn}$ [52]. They utilized the correlations between $L$ and $\delta R_{n p}$ within various models to predict the skin thicknesses of ${ }^{68} \mathrm{Ni}$ and ${ }^{132} \mathrm{Sn}$ and extrapolate $\delta R_{n p}=0.194 \pm 0.024 \mathrm{fm}$ for ${ }^{208} \mathrm{~Pb}$. In addition, the authors [53] obtain a value of $S_{0}=32.3 \pm 1.3 \mathrm{MeV}$ using the correlation between $L$ and $S_{0}$, calculated by the same models. The PDR symmetry energy constraint is shown as a dashed rectangle in Fig. 2 with the label PDR at the bottom of the box.

\section{THEORETICAL MODELS OF NUCLEI AND NUCLEONIC MATTER}

One of the outstanding problems in theoretical nuclear physics is to determine the role of three- and more- body forces in calculating the nuclear equation of state. In this section, we discuss calculations of the symmetry energy using representative theoretical models. We examine the range of calculated symmetry energies and neutron skin thickness predicted by calculations using phenomenological interactions and by calculations using microscopic and phenomenological two nucleon forces and phenomenological three- and morebody forces. We also show that the experimental constraints obtained in previous sections allow one to distinguish different interactions and verify the importance of three-neutron $(3 n)$ forces.

\section{A. Phenomenological models}

Many calculations employ effective, density-dependent nucleon-nucleon interaction of a Skyrme or Gogny type

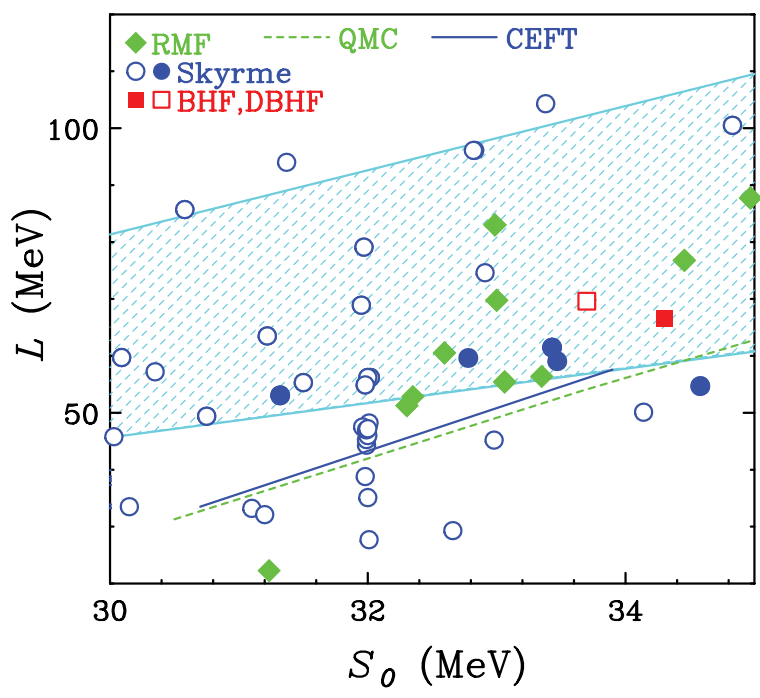

FIG. 3. (Color online) Symmetry energy correlations from different models (symbols). The dashed and solid lines represent the linear relationship between $L$ and $S_{0}$ in the QMC and CEFT models, respectively. The shaded region represents constraints obtained from the HIC experiment. The axes are the same as Fig. 2.

[15-17] or meson exchange interactions based on a relativistic mean-field model (RMF) Lagrangian approach [18,19]. The strength and ranges of the various terms in these phenomenological interactions are adjusted to describe nuclear properties, with little direct input from nucleon-nucleon scattering. One of the ultimate goals for studies of the symmetry energy should be to narrow the experimentally and theoretically allowed region in the $S_{0}-L$ plane.

The interactions utilized by these Skyrme, or RMF, approaches typically have a number of adjustable correlated parameters, which have not been adequately constrained by existing data, leading to a proliferation of different parameterizations. In Fig. 3, we show a wide range of possible $S_{0}-L$ correlations predicted by a selection of these phenomenological parameterizations. The open circles show predictions of the $S_{0}-L$ correlation for a set of Skyrme interactions used in Ref. [68]. The experimental constraints from HIC exclude some interactions.

In Ref. [20], Dutra et al. extended the set of constraints, usually utilized in the development of Skyrme parameterization, to 11 macroscopic conditions, originating from empirical properties of nuclear matter and experiments, and four microscopic constraints including density dependence of the effective mass and Landau parameters, to test the suitability of 240 Skyrme interactions. The combined effect of these constraints leaves only five Skyrme interactions that satisfied nearly all the constraints. The $L$ and $S_{0}$ values calculated with the selected interactions (solid circles) cluster along the lower boundaries of the experimentally allowed regions in Fig. 3.

\section{B. Microscopic models}

Microscopic approaches start from free two-body nucleonnucleon interactions $(\mathrm{NN})$ that reproduce nucleon-nucleon 
scattering and three-body interactions, which together with the two-body interactions, reproduce bound state properties of selected light nuclei [21,22]. The in-medium correlations to these interactions can then be calculated by many-body techniques, such as the Brueckner-Hartree-Fock (BHF) or its relativistic counterpart, the Dirac-Brueckner-Hartree-Fock (DBHF) (see, e.g., $[23,24]$ ). We note that most of the bulk of the relativistic effects typical of DBHF approaches can be associated with the class of three-body forces originating from virtual pair excitation.

The Quantum Monte Carlo (QMC) technique provides another way to include the main-body correlations and solve the many-body problem. This technique can tackle the problem exactly when the interactions are local in configuration space. However, it requires significant computational resources, making large nuclei impossible at present to compute. QMC calculations for nuclear matter have successfully demonstrated a strong correlation between the symmetry energy and its density dependence [25,26], shown as the dash line in Fig. 3.

Finally, chiral effective field theory (CEFT), with renormalization group (RG)-evolved interactions constrained by nucleon scattering data [27], has recently been used to calculate nuclear matter properties $[28,29]$. These calculations constrained the pressure of neutron matter at saturation densities within $\pm 25 \%$.

In Fig. 4 we plot several theoretical predictions of the density dependence of the pressure in pure neutron matter. The left panel shows results that include two-body potentials without inclusion of three-body neutron $(3 n)$ forces. The curves are calculated using the BHF approach with the Av18 potential [23], the QMC approach with the Av8' potential [26], and the CEFT approach [29]. In the right panel we demonstrate the effect of including $3 n$ forces and show results using the BHF approach with the Av18 + UIX three-body potential [23], the DBHF approach with the relativistic one-boson-

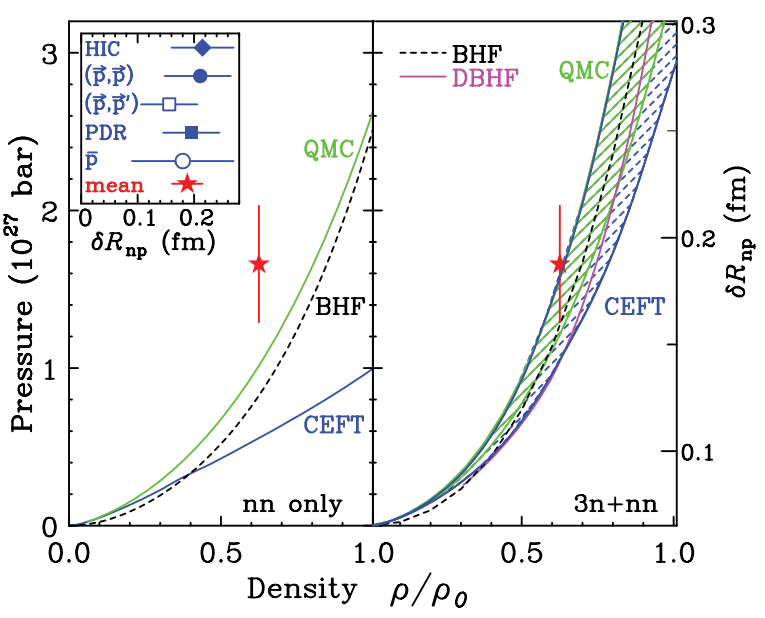

FIG. 4. (Color online) Density dependence of pressure in pure neutron matter as predicted in BHF, QMC, and CEFT models without (left panel) and with (right panel) $3 n$ forces. The inset in the left panel shows experimental data currently available on the neutron skin thickness in ${ }^{208} \mathrm{~Pb}$ and the red star indicates their weighted average. For more details see text. exchange Bonn B potential [24], the QMC approach with the $A \mathrm{v} 8^{\prime}+V_{2 \pi}^{\mathrm{PW}}+V_{m=150}^{R}$ potential (lower limit), and the $\mathrm{Av} 8^{\prime}+$ UIX potential (upper limit of the green shaded area) [26]. These limits are derived from the spread of predictions, calculated using different forms of the three-neutron force [26]. The lower and upper limit of the CEFT predictions reflect the theoretical error of the model $[28,29]$. Even though the calculations shown in the right panel of Fig. 4 have been calculated with different models using different forms of threebody potentials, all calculations lie within the uncertainties of the CEFT calculations (blue shaded region). Furthermore, the upper limits obtained for the QMC and CEFT approaches are almost identical. Figure 4 also demonstrates that individual contributions to observables are in general shifted between two-body neutron-neutron $(n n)$ and $3 n$ forces (and higherbody forces) by changing the RG resolution scale of the Hamiltonian. Consequently only the sum of all contributions is an observable. At low resolution scales (corresponding to the CEFT calculations), the size of $3 n$ contributions to neutron matter pressure is significantly larger than at higher RG resolution scales (corresponding to the QMC and BHF results). Even after the theoretical uncertainties are taken into account, the $3 n$ force significantly increases the pressure of neutron matter which affects the neutron skin thickness in ${ }^{208} \mathrm{~Pb}$.

These models can also be used to predict the correlation between $S_{0}$ and $L$. Like the Skyme interactions, predictions from the RMF models (solid diamonds) from Ref. [82] are scattered on the plane. The $S_{0}-L$ correlation predicted by BHF [23] and DBHF [24] are shown as squares. Both the QMC and CEFT models predict a linear dependence of $S_{0}$ and $L$ as shown by the green dash and blue solid lines in Fig. 3, respectively. Both lines are nearly the same and lie below the lower boundary of the experimentally preferred $S_{0}-L$ correlation, intersecting it only at higher $S_{0}>34 \mathrm{MeV}$. Many of the predicted values for $L$ lie near the lower boundary of the experimentally preferred $S_{0}-L$ region. The strong correlation from the QMC model has been recently combined with the constraints on the mass and radius of neutron stars to give $31.2 \mathrm{MeV}<S_{0}<34.3 \mathrm{MeV}$ and $36 \mathrm{MeV}<L<55 \mathrm{MeV}$ to $95 \%$ confidence level [57]. These constraints, derived from theoretical analyses of x-ray data from satellite observatories, are shown as a red dashed box labeled as $n$-star in Fig. 2 . The constraints tend to favor a weak density dependence of symmetry energy, barely overlapping with the lower bound of the boundaries determined from nuclear experiments. We note that the small extracted values of the radii of neutron stars depend on assumptions regarding the dynamics of $\mathrm{x}$-ray bursts and the emissivity of the stellar surface [83]. It will be an important scientific objective that both laboratory measurements and astrophysical observations can be described consistently with the same assumptions about the density dependence of the symmetry energy.

Neutron skin thickness values discussed in Sec. III are shown in the left-hand panel inset of Fig. 4. The ${ }^{208} \mathrm{~Pb}$ skin thicknesses determined from antiprotonic atom systematics, the weighted energy sum-rule of Pygmy Dipole resonances, the electric dipole polarizability and the proton elastic scattering experiment are plotted as open circle, solid square, open square 
and solid circle respectively. As discussed earlier, we adopt a minimum uncertainty of $\pm 0.05 \mathrm{fm}$. The skin thickness deduced from the symmetry energy constraints from nucleus-nucleus collisions using various Skyme forces is plotted as a solid diamond symbol in the inset. The red star in both panels depicts the weighted average of these values, using the relationship of Typel and Brown [59] between the pressure in neutron matter (left axis) at $\rho / \rho_{0}=0.625$ and the neutron skin for ${ }^{208} \mathrm{~Pb}$ (right axis). The theoretical uncertainties in the calculations are comparable to the experimental uncertainty. The current experimental uncertainty is a factor of two smaller than the designed error in the PREX experiment. It is probably unrealistic to expect measurements with smaller uncertainties. Nonetheless, this average value agrees better with calculations that include $3 n$ forces (right panel).

All the calculations lie lower than the experimental measurements of the skin thickness of ${ }^{208} \mathrm{~Pb}$, consistent with the low $L$ values plotted in Fig. 3. If future measurements do not shift the $S_{0}-L$ correlation to lower $L$, that may be an indication of insufficient current understanding of the three and higher-body forces.

\section{SUMMARY AND OUTLOOK}

The density dependence of the symmetry energy has wideranging ramifications in many branches of nuclear physics and astrophysics, motivating serious efforts to constrain it. We have shown that consistent results have been obtained from both nuclear structure measurements and from the measurement of heavy ion collisions. Our results provide a constraint centered around $\left(S_{0}, L\right) \sim(32.5,70) \mathrm{MeV}$ over the range of density between $0.3 \rho_{0} \leqslant \rho \leqslant \rho_{0}$. We also demonstrate that the neutron skin thickness for ${ }^{208} \mathrm{~Pb}$ of $\delta R_{n p}=0.18 \pm 0.027 \mathrm{fm}$ provide a constraint on the $3 n$ forces in macroscopic calculations of the density dependence of pure neutron matter. Both the symmetry energy constraints and neutron skin thickness measurements are consistent with each other.

Further refinements of theory and experiments and the extension of experimental data to higher and lower densities, particularly relevant for astrophysics, are needed. The skin thickness extracted in PREXII experiments [74] should have much smaller theoretical uncertainties. After ${ }^{208} \mathrm{~Pb}$ measurement, an additional measurement in ${ }^{48} \mathrm{Ca}$ would be very attractive because microscopic coupled cluster or no-core shell model calculations can closely relate $3 n$ forces to the skin thickness in ${ }^{48} \mathrm{Ca}$ [84]. Plans are underway to extend heavy-ion collision experiments $[35,85]$ to high energy to probe the symmetry energy at densities of $\rho \approx 2 \rho_{0}$ and beyond.

\section{ACKNOWLEDGMENTS}

The authors thank the organizers and participants of the International Symposium on Nuclear Symmetry Energy, 2011 (Nusym11) at Smith College where this project was conceived. The work is supported by the National Science Foundation, the Department of Energy and the Robert A. Welch Foundation, LANL LDRD and Open Supercomputing in the United States, the CPAN-MICINN in Spain, Fundação para a Ciência e a Tecnologia (FCT) in Portugal, and the Japan Society for the Promotion of Science.
[1] The Frontiers of Nuclear Science, Nuclear Science Advisory Committee's December 2007 Long Range Plan, http://science. energy.gov/np/nsac/.

[2] The Nuclear Physics European Collaboration Committee (NuPECC) Long Rang Plan, 2010, http://www.nupecc.org/ index.php?display $=\operatorname{lrp} 2010 /$ main.

[3] J. M. Lattimer and M. Prakash, Science 23, 536 (2004).

[4] The LIGO Scientific Collaboration, Nat. Phys, 7, 962 (2011).

[5] P. Danielewicz, R. Lacey, and W. G. Lynch, Science 298, 1592 (2002).

[6] B. A. Brown, Phys. Rev. Lett. 85, 5296 (2000).

[7] H. A. Bethe and R. F. Bacher, Rev. Mod. Phys. 8, 82 (1936).

[8] C. F. von Weizäcker and Z. Fur, Physik 96, 431 (1935).

[9] P. Möller, W. D. Myers, H. Sagawa, and S. Yoshida, Phys. Rev. Lett. 108, 052501 (2012).

[10] C. J. Horowitz and J. Piekarewicz, Phys. Rev. Lett. 86, 5647 (2001).

[11] S. J. Pollock, E. N. Fortson, and L. Wilets, Phys. Rev. C 46, 2587 (1992).

[12] S. J. Pollock and M. C. Welliver, Phys. Lett. B 464, 177 (1999).

[13] B. A. Brown, A. Derevianko, and V. V. Flambaum, Phys. Rev. C 79, 035501 (2009).

[14] K. Tsigutkin, D. Dounas-Frazer, A. Family, J. E. Stalnaker, V. V. Yashchuk, and D. Budker, Phys. Rev. A 81, 032114 (2010).

[15] D. Vautherin and D. M. Brink, Phys. Rev. C 5, 626 (1972).
[16] J. R. Stone and P.-G. Reinhard, Prog. Part. Nucl. Phys. 58, 587 (2007).

[17] J. Decharge and D. Gogny, Phys. Rev. 21, 1568 (1980).

[18] B. D. Serot and J. D. Walecka, Adv. Nucl. Phys. 16, 1 (1986); Int. J. Mod. Phys. E 6, 515 (1997).

[19] J. Meng, H.Toki, S. G. Zhou, S. Q. Zhang, W. H. Long, and L. S. Geng, Prog. Part. Nucl. Phys. 57, 470 (2006).

[20] M. Dutra, O. Lourenco, J. S. Sa Martins, A. Delfino, J. R. Stone, and P. D. Steveson, Phys. Rev. C 85, 035201 (2012).

[21] S. C. Pieper, V. R. Pandharipande, R. B. Wiringa, and J. Carlson, Phys. Rev. C 64, 014001 (2001).

[22] S. C. Pieper, Nucl. Phys. A 751, 516 (2005).

[23] I. Vidana, C. Providencia, A. Polls, and A. Rios, Phys. Rev. C 80, 045806 (2009).

[24] F. Sammarruca, arXiv:1111.0695; Int. J. Phys. E 19, 1259 (2010).

[25] S. Gandolfi, A. Y. Illarionov, S. Fantoni, J. C. Miller, F. Pederiva, and K. E. Schmidt, Mon. Not. R. Astron. Soc. 404, L35 (2010).

[26] S. Gandolfi, J. Carlson, and S. Reddy, Phys. Rev. C 85, 032801(R) (2012).

[27] S. K. Bogner, R. J. Furnstahl, and A. Schwenk, Prog. Part. Nucl. Phys. 65, 94 (2010).

[28] K. Hebeler and A. Schwenk, Phys. Rev. C 82, 014314 (2010).

[29] K. Hebeler, J. M. Lattimer, C. J. Pethick, and A. Schwenk, Phys. Rev. Lett. 105, 161102 (2010). 
[30] M. B. Tsang, Y. Zhang, P. Danielewicz, M. Famiano, Z. Li, W. G. Lynch, and A. W. Steiner, Phys. Rev. Lett. 102, 122701 (2009).

[31] W. G. Lynch, M. B. Tsang, Y. Zhanga, P. Danielewicz, M. Famiano, Z. Li, and A. W. Steiner, Prog. Part. Nucl. Phys. 62, 427 (2009).

[32] H. S. Xu, M. B. Tsang, T. X. Liu, X. D. Liu, W. G. Lynch, W. P. Tan, G. Verde, L. Beaulieu, B. Davin, Y. Larochelle, T. Lefort, R. T. de Souza, R. Yanez, V. E. Viola, R. J. Charity, and L. G. Sobotka, Phys. Rev. Lett. 85, 716 (2000).

[33] M. B. Tsang, T. X. Liu, L. Shi, P. Danielewicz, C. K. Gelbke, X. D. Liu, W. G. Lynch, W. P. Tan, G. Verde, A. Wagner, H. S. $\mathrm{Xu}$, W. A. Friedman, L. Beaulieu, B. Davin, R. T. de Souza, Y. Larochelle, T. Lefort, R. Yanez, V. E. Viola Jr., R. J. Charity, and L. G. Sobotka, Phys. Rev. Lett. 92, 062701 (2004).

[34] M. Famiano, T. Liu, W. G. Lynch, M. Mocko, A. M. Rogers, M. B. Tsang, M. S. Wallace, R. J. Charity, S. Komarov, D. G. Sarantites, L. G. Sobotka, and G. Verde, Phys. Rev. Lett. 97, 052701 (2006).

[35] P. Russotto, P. Z. Wu, M. Zoric, M. Chartier, Y. Leifels, R. C. Lemmon, Q. Li, J. Lukasik, A. Pagano, P. Pawlowski, and W. Trautmann, Phys. Lett. B 697, 471 (2011).

[36] Z. Kohley, L. W. May, S. Wuenschel, A. Bonasera, K. Hagel, R. Tripathi, R. Wada, G. A. Souliotis, D. V. Shetty, S. Galanopoulos, M. Mehlman, W. B. Smith, S. N. Soisson, B. C. Stein, and S. J. Yennello, Phys. Rev. C 82, 064601 (2010).

[37] Z. Kohley, L. W. May, S. Wuenschel, M. Colonna, M. Di Toro, M. Zielinska-Pfabe, K. Hagel, R. Tripathi, A. Bonasera, G. A. Souliotis, D. V. Shetty, S. Galanopoulos, M. Mehlman, W. B. Smith, S. N. Soisson, B. C. Stein, and S. J. Yennello, Phys. Rev. C 83, 044601 (2011).

[38] M. B. Tsang, C. K. Gelbke, X. D. Liu, W. G. Lynch, W. P. Tan, G. Verde, H. S. Xu, W. A. Friedman, R. Donangelo, S. R. Souza, C. B. Das, S. D. Gupta, and D. Zhabinsky, Phys. Rev. C 64 , 054615 (2001).

[39] T. X. Liu, W. G. Lynch, M. B. Tsang, X. D. Liu, R. Shomin, W. P. Tan, G. Verde, A. Wagner, H. F. Xi, H. S. Xu, B. Davin, Y. Larochelle, R. T. de Souza, R. J. Charity, and L. G. Sobotka, Phys. Rev. C 76, 034603 (2007).

[40] Z. Y. Sun, M. B. Tsang, W. G. Lynch, G. Verde, F. Amorini, L. Andronenko, M. Andronenko, G. Cardella, M. Chatterje, P. Danielewicz, E. De Filippo, P. Dinh, E. Galichet, E. Geraci, H. Hua, E. La Guidara, G. Lanzalone, H. Liu, F. Lu, S. Lukyanov, C. Maiolino, A. Pagano, S. Piantelli, M. Papa, S. Pirrone, G. Politi, F. Porto, F. Rizzo, P. Russotto, D. Santonocito, and Y. X. Zhang, Phys. Rev. C 82, 051603(R) (2010).

[41] M. S. Antony, A. Pape, and J. Britz, At. Data Nucl. Data Tables 66, 1 (1997).

[42] P. Danielewicz and J. Lee, AIP Conf. Proc. 1423, 29 (2012).

[43] S. Abrahamyan, Z. Ahmed, H. Albataineh, K. Aniol, D. S. Armstrong, W. Armstrong, T. Averett, B. Babineau, A. Barbieri, V. Bellini et al., Phys. Rev. Lett. 108, 112502 (2012).

[44] J. Zenihiro, H. Sakaguchi, T. Murakami, M. Yosoi, Y. Yasuda, S. Terashima, Y. Iwao, H. Takeda, M. Itoh, H. P. Yoshida, and M. Uchida, Phys. Rev. C 82, 044611 (2010); J. Zenihiro, Ph.D. Thesis, Kyoto University, 2011.

[45] S. Terashima, H. Sakaguchi, H. Takeda, T. Ishikawa, M. Itoh, T. Kawabata, T. Murakami, M. Uchida, Y. Yasuda, M. Yosoi, J. Zenihiro, H. P. Yoshida, T. Noro, T. Ishida, S. Asaji, and T. Yonemura, Phys. Rev. C 77, 024317 (2008).
[46] T. Suzuki, H. Geissel, O. Bochkarev, L. Chulkov, M. Golovkov, D. Hirata, H. Irnich, Z. Janas, H. Keller, T. Kobayashi, G. Kraus, G. Münzenberg, S. Neumaier, F. Nickel, A. Ozawa1, A. Piechaczeck, E. Roeckl, W. Schwab, K. Sümmerer, K. Yoshida, and I. Tanihata, Phys. Rev. Lett. 75, 3241 (1995).

[47] A. Trzcińska, J. Jastrzębski, P. Lubiński, F. J. Hartmann, R. Schmidt, T. von Egidy, and B. Kłos, Phys. Rev. Lett. 87, 082501 (2001).

[48] B. Kłos, A. Trzcińska, J. Jastrzębski, T. Czosnyka, M. Kisieliński, P. Lubiński, P. Napiorkowski, L. Pieńkowski, F. J. Hartmann, B. Ketzer, P. Ring, R. Schmidt, T. von Egidy, R. Smolańczuk, S. Wycech, K. Gulda, W. Kurcewicz, E. Widmann, and B. A. Brown, Phys. Rev. C 76, 014311 (2007).

[49] M. Centelles, X. Roca-Maza, X. Viñas, and M. Warda, Phys. Rev. Lett. 102, 122502 (2009); Phys. Rev. C 80, 024316 (2009).

[50] A. Tamii, I. Poltoratska, P. von Neumann-Cosel, Y. Fujita, T. Adachi, C. A. Bertulani, J. Carter, M. Dozono, H. Fujita, K. Fujita et al., Phys. Rev. Lett. 107, 062502 (2011).

[51] L. Trippa, G. Colo, and E. Vigezzi, Phys. Rev. C 77, 061304(R) (2008).

[52] A. Klimkiewicz, N. Paar, P. Adrich, M. Fallot, K. Boretzky, T. Aumann, D. Cortina-Gil, U. Datta Pramanik, Th. W. Elze, H. Emling, H. Geissel, M. Hellström, K. L. Jones, J. V. Kratz, R. Kulessa, C. Nociforo, R. Palit, H. Simon, G. Surówka, K. Sümmerer, D. Vretenar, and W. Waluś, Phys. Rev. C 76, 051603(R) (2007).

[53] A. Carbone, G. Colò, A. Bracco, L.-G. Cao, P. F. Bortignon, F. Camera, and O. Wieland, Phys. Rev. C 81, 041301 (2010).

[54] O. Wieland A. Bracco, F. Camera, G. Benzoni, N. Blasi, S. Brambilla, F. C. L. Crespi, S. Leoni, B. Million, R. Nicolini et al., Phys. Rev. Lett. 102, 092502 (2009).

[55] B.-A. Li, L.-W. Chen, and C. M. Ko, Phys. Rep. 464, 113 (2008).

[56] Y. Zhang, D. D. S. Coupland, P. Danielewicz, Z. Li, H. Liu, F. Lu, W. G. Lynch, and M. B. Tsang, Phys. Rev. C 85, 024602 (2012)

[57] A. W. Steiner and S. Gandolfi, Phys. Rev. Lett. 108, 081102 (2012).

[58] J. M. Lattimer and Y. Lim, arXiv:1203.4286.

[59] S. Typel and B. A. Brown, Phys. Rev. C 64, 027302 (2001).

[60] A. W. Steiner, M. Prakash, J. M. Lattimer, and P. J. Ellis, Phys. Rep. 411, 325 (2005).

[61] P. Danielewicz and J. Lee, Nucl. Phys. A 818, 36 (2009).

[62] C. J. Horowitz and A. Schwenk, Nucl. Phys. A 776, 55 (2006).

[63] J. B. Natowitz, G. Röpke, S. Typel, D. Blaschke, A. Bonasera, K. Hagel, T. Klähn, S. Kowalski, L. Qin, S. Shlomo, R. Wada, and H. H. Wolter, Phys. Rev. Lett. 104, 202501 (2010).

[64] S. R. Souza, M. B. Tsang, B. V. Carlson, R. Donangelo, W. G. Lynch, and A. W. Steiner, Phys. Rev. C 80, 041602(R) (2009), and references therein.

[65] C. Fuchs, Prog. Part. Nucl. Phys. 1, 56 (2006).

[66] J. Rizzo, M. Colonna, V. Baran, M. Di Toro, H. H. Wolter, and M. Zielinska-Pfabe, Nucl. Phys. A 806, 79 (2008).

[67] W. D. Myers and W. J. Swiatecki, Ann. Phys. 55, 395 (1969); 84, 186 (1974).

[68] P. Danielewicz, Nucl. Phys. A 727, 233 (2003).

[69] M. S. Antony, A. Pape, and J. Britz, At. Data Nucl. Data Tables 66, 1 (1997).

[70] G. Fricke, C. Bernhardt, K. Heilig, L. A. Schaller, L. Schellenberg, E. B. Shera, and C. W. Dejager, At. Data Nucl. Data Tables 60, 177 (1995). 
[71] B. A. Brown, G. Shen, G. C. Hillhouse, J. Meng, and A. Trzcińska, Phys. Rev. C 76, 034305 (2007).

[72] C. J. Horowitz, Phys. Rev. C 57, 3430 (1998); Eur. Phys. J. A 30, 303 (2006).

[73] C. Horowitz, S. J. Pollock, P. A. Souder, and R. Michaels, Phys. Rev. C 63, 025501 (2001).

[74] P. A. Souder, R. Holmes, Chun-Min Jen, L. Zana, Z. Ahmed, A. Rakhman, E. Cisbani, S. Frullani, F. Garibaldi, F. Meddi et al., PREX II experimental proposal to Jefferson Laboratory, PAC38, http://hallaweb.jlab.org/parity/prex/prexII.pdf.

[75] N. L. Rodning, L. D. Knutson, W. G. Lynch, and M. B. Tsang, Phys. Rev. Lett. 49, 909 (1982).

[76] B. L. Berman and S. C. Fultz, Rev. Mod. Phys. 47, 713 (1975).

[77] S. Stringari and E. Lipparini, Phys. Lett. B 117, 141 (1982).
[78] H. Krivine, J. Treiner, and O. Bohigas, Nucl. Phys. A 336, 155 (1980).

[79] J. Piekarewicz, Phys. Rev. C 83, 034319 (2011).

[80] P.-G. Reinhard and W. Nazarewicz, Phys. Rev. C 81, 051303(R) (2010).

[81] J. Piekarewicz, B. K. Agrawal, G. Colò, W. Nazarewicz, N. Paar, P.-G. Reinhard, X. Roca-Maza, and D. Vretenar, Phys. Rev. C 85, 041302(R) (2012).

[82] X. Roca-Maza, M. Centelles, X. Viñas, and M. Warda, Phys. Rev. Lett. 106, 252501 (2011).

[83] A. W. Steiner, J. M. Lattimer, and E. F. Brown, Astrophys. J. 722, 33 (2010).

[84] S. Ban, C. J. Horowitz, and R. Michaels, J. Phys. G: Nucl. Part. Phys. 39, 015104 (2012).

[85] Symmetry Energy Project, http://groups.nscl.msu.edu/hira/ sep.htm. 